\title{
OTIOLTOMTS
}

Revista de economía, empresa y sociedad

Dosier sobre economía colaborativa (y II)

Nuevas estrategias y dimensiones alternativas de la economía de plataforma

Coordinador: Lluís Alfons Garay Tamajón

SUB IUDICE

\section{Plataformas digitales en el sector de los VTG}

\section{Gabriel Doménech Pascual}

Universitat de València

RESUMEN El del transporte urbano de pasajeros con vehículos turismo (taxi y VTC) es, seguramente, el sector donde la irrupción de la economía colaborativa -o, si se prefiere decir así, las plataformas digitales- ha dado lugar a controversias más encendidas, medidas regulatorias más cuestionables y un mayor número de pleitos. En el presente trabajo se hace un breve repaso de las cuestiones regulatorias más candentes que se han planteado, haciendo hincapié en las que todavía están pendientes de resolución en un sentido que no parece fácil avizorar.

PALABRAS CLAVE economía colaborativa; plataformas digitales; taxi; transporte urbano de pasajeros.

\section{Digital platforms in the vehicle for hire sector}

ABSTRACT The sector of urban passenger transport with tourism vehicles (taxis and vehicles for hire) is, surely, the one where the appearance of the sharing economy-or, we can choose to say, the digital platforms- has given rise to more heated controversies, more questionable regulatory measures and a greater number of disputes. In the work presented herein, a brief review is set out of the latest regulatory issues which have been outlined here, highlighting those which are still awaiting resolution, in a sense which is seemingly not easy to perceive..

KEYWORDS sharing economy; digital platforms; taxis; urban passenger transport 


\section{La irrupción de las plataformas digitales en el sector de los VTC}

Durante los últimos años, varios sectores de la economía han experimentado una fuerte sacudida como consecuencia de la aparición de plataformas digitales (v. gr., Uber, Cabify, BlaBlaCar, Deliveroo, Glovo y AirBnB). En algunos de estos sectores, dicha aparición se ha visto envuelta en una intensa polémica, que ha tenido su reflejo jurídico en forma de un alud de medidas regulatorias y pleitos. Las disputas han sido especialmente fuertes allí donde existía una densa regulación y las autoridades reguladoras estaban en buena medida capturadas por los operadores ya establecidos.

El sector del transporte urbano de pasajeros con turismos es un buen ejemplo. Los taxis y los llamados vehículos turismo con conductor (VTC) se reparten este mercado. Ambos han estado sometidos a numerosas restricciones regulatorias, entre las cuales destacan las siguientes:

1) Para realizar esta actividad se requiere la obtención de una autorización administrativa previa, cuyo número -y, a la postre, el de los vehículos con los que se puede prestar el servicio- ha sido «artificialmente» limitado por las autoridades reguladoras.

2) Este mercado está fragmentado territorialmente. Los servicios de taxi solo pueden iniciarse en el término del municipio que haya otorgado la correspondiente licencia, salvo excepciones. Los VTC, por su parte, solo pueden prestar servicios habitualmente dentro del territorio de la comunidad autónoma en que se encuentre domiciliada la autorización en que se amparan.

Las diferencias regulatorias más significativas entre ambos tipos de vehículos son las siguientes:

- Los precios y otras condiciones (v. gr., horarios y calendarios) de los servicios prestados por los taxis están regulados.

- El número de autorizaciones de taxi de las que una misma persona puede ser titular suele limitarse.

- Los taxis pueden tomar pasajeros en paradas, directamente en la calle a solicitud de estos, o contratar sus servicios con antelación. Los VTC, en cambio, únicamente pueden prestar servicios previamente contratados.

- Durante el periodo que medió entre la Ley 25/2009 y el Real Decreto 1057/2015 no existieron restricciones cuantitativas para solicitar licencias de VTC. Esta ventana temporal de libertad de entrada propició un considerable incremento de la flota de VTC. Si en 2010 había casi 3.000 licencias, en 2021 hay 16.911 licencias en España (frente a las 64.315 de taxi).

En este contexto normativo, la irrupción de plataformas como Uber y Cabify ha hecho posible: 1) reducir drásticamente las asimetrías informativas y los costes de transacción que conlleva precontratar un VTC; 2) generar considerables economías de escala y de red; y 3) aprovechar el enorme incremento de licencias VTC para prestar de manera muy eficiente y rentable un gran volumen de servicios de transporte.

De resultas de ello, muchas de las regulaciones arriba descritas se han vuelto desproporcionadas, por cuanto los fallos del mercado que trataban de combatir pueden ser eliminados o paliados ahora de manera mucho más eficiente a través de las referidas plataformas digitales. La experiencia de otros países, donde se ha liberalizado el sector, permite estimar que la eliminación de varias de esas restricciones incrementaría el bienestar social, especialmente de los usuarios.

Sirvan dos ejemplos. El primero es el de la limitación del número de vehículos. El principal argumento aducido para establecerla es el de las externalidades -polución ambiental y congestión del tráfico- que estos generan. Pero este argumento no es atendible, por varias razones.

a) Si se quiere combatir esos efectos negativos, lo que habría que hacer es reducir el número global de turismos que circulan por nuestras ciudades, y no solo el de taxis o VTC en particular.

b) Aquella limitación puede resultar inútil o incluso contraproducente para alcanzar dicha reducción. No está en absoluto claro que una restricción tal de la oferta de taxis y VTC vaya a repercutir positivamente sobre el medio ambiente y la fluidez del tráfico. Es posible que algunos individuos que viajarían en ellos si la oferta fuese mayor recurran a vehículos privados y que, por ende, tales externalidades acaben agravándose. 
c) Abundantes estudios han puesto de manifiesto que el establecimiento de un impuesto que grave el acceso al centro urbano permite lograr dichos objetivos de manera mucho más eficiente. Esta alternativa es, desde luego, mucho más respetuosa con la libertad y la igualdad, ya que deja a todos los ciudadanos la posibilidad de transitar por allí y, en principio, se aplica igualmente a todos los que desarrollan esta actividad.

El segundo es el de la propia dualidad regulatoria existente entre taxis y VTC. A la vista de que hoy en día precontratar un VTC a través de una plataforma digital resulta más fácil y eficiente que tomar un taxi en una parada o «levantando una mano", ¿qué sentido tiene seguir manteniendo esa dualidad? ¿Qué se gana con ella?

\section{Resistencia al cambio}

Uno podría pensar que lo que deberían hacer las autoridades competentes es revisar esas restricciones regulatorias, eliminar las que se hayan vuelto obsoletas y permitir que las plataformas digitales operen en este sector sin más limitaciones que las estrictamente necesarias y no excesivas para corregir los fallos del mercado todavía subsistentes.

Sin embargo, no es eso lo que ha ocurrido. Más bien, todo lo contrario. En líneas generales, tales autoridades han adoptado toda una batería de medidas dirigidas a intensificar dichas restricciones y proteger a los taxis frente a la competencia que suponen los VTC.

Esa reacción se explica en gran medida por dos factores. El primero -y, probablemente, más determinante- es que esas autoridades llevan décadas capturadas por el gremio de los taxistas, que ha logrado ejercer sobre ellas una enorme presión, con resultados muy exitosos. El segundo factor es que, en la mayoría de las comunidades autónomas, la presencia de los VTC es todavía muy escasa, lo que hace que los titulares de sus licencias, sus trabajadores y sus usuarios tengan una capacidad muy reducida de influir sobre las autoridades y contrarrestar las presiones del lobby del taxi. Resulta muy significativo que en ninguna de las cuatro comunidades autónomas donde la ratio VTC/taxis es mayor (Madrid, La Rioja, Cantabria y Andalucía) se hayan adoptado «medidas hostiles» contra los primeros. ${ }^{1}$

\section{La reacción de las autoridades reguladoras}

\subsection{Reacción inicial: contención}

En líneas generales, esta reacción inicial consistió en frenar y contener la expansión de los VTC, así como proteger a los taxistas frente a la creciente competencia que los primeros representaban.

En efecto, por de pronto, prácticamente todas las comunidades autónomas denegaron sistemáticamente las licencias VTC solicitadas durante el periodo de liberalización, 2009-2015, lo que obligó a los solicitantes a acudir a los tribunales para que estos les reconocieran su derecho a obtenerlas.

En segundo lugar, tanto las comunidades autónomas como el Estado aprobaron varias medidas encaminadas a entorpecer la actividad de los VTC, algunas de las cuales fueron consideradas contrarias a derecho por los tribunales. Por ejemplo, el Decreto Ley catalán 5/2017, entre otras cosas, impuso una moratoria de dos años para la transmisión de autorizaciones de VTC y creó un registro electrónico de comunicaciones de los servicios de VTC con el fin de someter a control administrativo cada uno de los prestados por los mismos. La referida moratoria

1. Según datos del Ministerio de Fomento, a 1 de marzo de 2021, en Andalucía había 9.195 taxis y 2.900 VTC. En Madrid, 15.714 taxis y 8.023 VTC. En Cantabria, 512 taxis y 143 VTC. Y, en La Rioja, 166 taxis y 83 VTC. 
fue declarada inconstitucional por la Sentencia del Tribunal Constitucional 105/2018. El Gobierno central, por su parte, estableció, en virtud del Real Decreto 1076/2017, una moratoria y un registro electrónico prácticamente idénticos, medidas que fueron consideradas ilegales y anuladas por la Sentencia del Tribunal Supremo de 10 de marzo de 2020 (ECLI:ES:TS:2020:732).

Aquí pueden mencionarse, también, las prohibiciones o limitaciones de la posibilidad de circular (v. gr., por el centro urbano) que algunos ayuntamientos (v. gr., de Madrid, Córdoba y Granada) han impuesto solo a los VTC, pero no a los taxis, y que varios juzgados han considerado discriminatorias.

Finalmente, se han otorgado beneficios cuestionables a los taxistas. Aquí destacan los programas de rescate de licencias. En lugar de incrementar la oferta, a fin de intensificar la competencia y mejorar los servicios en beneficio de los usuarios, algunos ayuntamientos (v. gr., los de Sevilla y Santa Cruz de Tenerife) han optado por restringirla todavía más, pagando cantidades considerables a los taxistas que voluntariamente deciden extinguir sus licencias.

\subsection{Reacción posterior: penalty y expulsión}

En una segunda fase, y como respuesta a la creciente presión del lobby del taxi, se ha tratado de expulsar a los VTC del mercado.

\subsubsection{La ordenanza de la Área Metropolitana de Barcelona de 2018}

El primer hito en este sentido lo constituye la ordenanza de la Área Metropolitana de Barcelona (AMB) de 26 de junio de 2018, que vino a dejar sin efectos en su territorio las autorizaciones de VTC otorgadas después de 2009, lo cual suponía de facto expropiarlas. Esta ordenanza incurría en varias ilegalidades flagrantes y, por esta razón, fue suspendida cautelarmente por el Auto del Tribunal Superior de Justicia de Cataluña de 26 de julio de 2018.

\subsubsection{El Real Decreto Ley $13 / 2018$}

En virtud de esta norma, el Gobierno central dispone que las licencias VTC «habilitarán exclusivamente para realizar transporte interurbano de viajeros», entendiéndose por tal aquel cuyo «recorrido rebase el territorio de un único término municipal o zona de prestación conjunta de servicios de transporte público urbano». Es decir, estas licencias ya no permiten realizar transportes urbanos, lo que prácticamente les priva de cualquier utilidad, puesto que la abrumadora mayoría de las carreras que actualmente hacen los VTC transcurren íntegramente por un único término municipal o zona de prestación conjunta de servicios de transporte público urbano.

Adicionalmente, se restringe también la prestación de servicios interurbanos, pues se establece, como regla general, que estos «deberán iniciarse en el territorio de la comunidad autónoma en que se encuentre domiciliada la correspondiente autorización».

Estas restricciones son tan intensas que abocan a la desaparición a la práctica totalidad de los VTC. Consciente de que esta regulación constituye una expropiación de las correspondientes licencias, el Gobierno prevé como «indemnización» que, durante los cuatro años posteriores a la entrada en vigor del Real Decreto Ley 13/2018, sus titulares «podrán continuar prestando servicios de ámbito urbano», plazo que puede extenderse hasta los seis años, si concurren determinadas circunstancias. Cabe poner en duda, obviamente, que esta compensación baste para dejar «indemnes» a los expropiados.

El decreto ley también «habilita» a las comunidades autónomas para regular las condiciones de explotación de los servicios "cuyo itinerario se desarrolle íntegramente en su respectivo ámbito territorial», con arreglo a ciertos límites.

Además, establece que «los servicios de transporte prestados en el ámbito urbano por los titulares de dichas autorizaciones quedarán sujetos a todas las determinaciones y limitaciones que establezca el órgano competente en materia de transporte urbano en el ejercicio de sus competencias sobre utilización del dominio público 
viario, gestión del tráfico urbano, protección del medio ambiente y prevención de la contaminación atmosférica; especialmente en materia de estacionamiento, horarios y calendarios de servicio o restricciones a la circulación por razones de contaminación atmosférica». Parece, pues, que se permite a las administraciones autonómicas y locales imponer a los VTC condiciones de prestación de servicio hasta ahora inexistentes, lo que, obviamente, disminuiría todavía más la rentabilidad de su actividad y haría aún más cuestionable la compensación de 4-6 años prevista.

Este real decreto ley incurre, en nuestra opinión, en varias infracciones de la Constitución española. ${ }^{2}$ No sería de extrañar, por ello, que el Tribunal Constitucional acabara pronunciándose al respecto.

\subsubsection{Disposiciones autonómicas}

Varias comunidades autónomas han publicado sendas disposiciones por las cuales imponen a los VTC, principalmente, tres restricciones, que prácticamente les impiden seguir operando como lo venían haciendo: 1) la necesidad de que entre la contratación del servicio y su prestación transcurra un periodo mínimo de tiempo (que oscila entre los 15 y los 30 minutos); 2) la prohibición de mostrar a los potenciales usuarios la geolocalización de los vehículos; y 3) la obligación de que los vehículos permanezcan estacionados fuera de las vías públicas cuando no estén prestando el servicio, o la prohibición de circular por estas vías para propiciar la captación de clientes, o incluso la prohibición de captar clientela en zonas de concentración de la demanda. ${ }^{3}$

La conformidad a derecho de estas restricciones es ciertamente dudosa. El Consell de Garanties Estatutàries de la Generalitat de Catalunya ha considerado que el referido periodo de contratación supone una restricción desproporcionada y, por lo tanto, ilícita, de la libertad de empresa reconocida en el artículo 38 de la Constitución. ${ }^{4}$ Por una razón análoga, el Tribunal Superior de Justicia de Cataluña ha suspendido cautelarmente dos disposiciones de una nueva ordenanza de la AMB, que elevaban el periodo mínimo de contratación hasta los 60 minutos y obligaban a los VTC a inscribirse en un registro municipal. ${ }^{5} \mathrm{El}$ Tribunal Superior de Justicia del País Vasco, por su parte, ha suspendido cautelarmente las disposiciones relativas al periodo mínimo de precontratación y a la geolocalización de los vehículos contenidas en el correspondiente decreto del Gobierno Vasco. ${ }^{6}$

\subsection{Comunidades autónomas no hostiles}

Como ya hemos señalado, las comunidades autónomas donde mayor es la ratio de VTC/taxis (Madrid, La Rioja, Cantabria y Andalucía) se han abstenido de adoptar medidas significativamente «hostiles» contra los primeros. Es más, los partidos políticos gobernantes en las comunidades donde más VTC hay en términos absolutos (Madrid y Andalucía) han manifestado públicamente su voluntad de establecer una regulación que garantice una cierta competencia entre ambos tipos de vehículos. La reciente convocatoria de elecciones a la Asamblea de Madrid, sin embargo, deja la referida regulación en el aire. Ya veremos cómo evolucionan los acontecimientos, pues los partidos de la oposición han sido los grandes impulsores de la solución consistente en «expulsar» a los VTC del mercado.

2. Véase Doménech Pascual (2019).

3. Véanse el Decreto Ley catalán 4/2019, de 29 de enero; el Decreto Ley balear 1/2019, de 22 de febrero; el Decreto Ley aragonés de 7/2019, de 12 de marzo; el Decreto Ley valenciano 4/2019, de 29 de marzo; y el Decreto vasco 200/2019, de 17 de diciembre.

4. Dictamen 2/2019, de 22 de febrero.

5. Auto de 9 de julio de 2019.

6. 6 Auto de 10 de febrero de 2020 (ECLI:ES:TSJPV:2020:60A). 


\section{El turno de los tribunales, perspectivas de futuro}

La aparición de plataformas digitales en este y otros sectores se ha visto envuelta en un reguero de pleitos, con resultados «mixtos». Los partidarios de liberalizar el sector han obtenido muchas victorias parciales -como las que se han ido reseñando a lo largo de este artículo-, pero también grandes derrotas, como la que supuso la Sentencia del Tribunal Supremo de 4 de junio de 2018 (ECLI:ES:TS:2018:1913), que avaló casi todas las restricciones existentes en el mercado de los VTC y, muy especialmente, las cuantitativas.

El futuro del sector depende en gran medida del resultado de numerosos pleitos que actualmente están pendientes de resolución y en los que los tribunales han de pronunciarse sobre algunas de las piezas clave de la regulación vigente, como la conformidad del Real Decreto Ley 13/2018 con la Constitución y el derecho de la Unión Europea.

Hay una gran incertidumbre acerca de cómo se van a resolver esos litigios. Por un lado, existen razones más que sobradas para considerar inconstitucionales muchas de las medidas adoptadas con el fin de dificultar o impedir la actividad de los VTC. Pero, por otro lado, hay que tener en cuenta que, tradicionalmente, nuestros tribunales han sido extremadamente deferentes con el legislador a la hora de enjuiciar restricciones de la libertad de empresa, hasta el punto de que nunca se ha declarado inconstitucional una norma con rango de ley por este motivo. Puede afirmarse que la libertad de empresa prevista en el artículo 38 de la Constitución no es en nuestro ordenamiento jurídico, de facto, un verdadero derecho constitucional, oponible frente al legislador.

Además, debe notarse que a los titulares de licencias VTC tampoco les interesa que se eliminen todas aquellas restricciones injustificadas y, en particular, la relativa al número de vehículos que en este sector puede operar, pues ello reduciría prácticamente a cero el valor de tales licencias. Lo que les interesa, sobre todo, es que se supriman otras limitaciones que entorpecen su libertad de prestación de servicios o, al menos, que se les permita seguir operando en este mercado como venían haciéndolo antes de la aprobación del Real Decreto Ley 13/2018.

En fin, resulta difícil avizorar cómo evolucionará este sector en el futuro. A la incerteza existente respecto del sentido en el que se van a resolver las dispuestas judiciales y políticas pendientes, se añade la posibilidad de que surjan innovaciones tecnológicas que transformen significativamente las condiciones de este mercado. Sea como fuere, cuesta imaginar que, por mor de una decisión política -económica y jurídicamente injustificada-, en dos años vaya a desaparecer un sistema productivo que ha acreditado sobradamente su eficiencia, que mueve muchos millones de euros y que da trabajo directo a unas 20.000 personas.

\section{Referencias bibliográficas}

AUTORITAT CATALANA DE LA COMPETĖNCIA (2018). Estudio sobre el sector del transporte de viajeros en vehículos de hasta nueve plazas: el taxi y los vehículos de alquiler con conductor (ES 15/2018).

DOMÉNECH PASCUAL, G. (2017). «La regulación de la economía colaborativa en el sector del taxi y los VTC». En MONTERO PASCUAL, J.J. (ed.): La regulación de la economía colaborativa. Airbnb, BlaBlaCar, Uber y otras plataformas. Valencia, Tirant lo Blanch, pág. 351-401.

DOMÉNECH PASCUAL, G. (2019). «Penalty y expulsión. La economía de las plataformas digitales en el sector de los taxis y los VTC». En ORTÍ VALLEJO, A.; RUBIO GIMENO, G. (eds.): Propuestas de regulación de las plataformas de economía colaborativa. Perspectivas general y sectoriales. Cizur Menor, Aranzadi.

GONZÁLEZ CABRERA, I. (2020). «Taxis y VTCS/PHVS (private hire vehicles) en España y en el Reino Unido. Una visión crítica de las regulaciones desarrolladas en las áreas metropolitanas de Barcelona y de Londres». Revista General de Derecho de los Sectores Regulados, núm. 5.

GÓRRIZ LÓPEZ, C. (2019). "Taxi vs. Uber: de la competencia desleal al arrendamiento de vehículo con conductor». Revista de Derecho Mercantil, núm. 311.

TORNOS MAS, J. (2019). Dret i innovación tecnológica. El cas Uber versus Taxi. Barcelona, Acadèmia de Jurisprudència i Legislació de Catalunya. 
Cita recomendada: DOMÉNECH, Gabriel. Plataformas digitales en el sector de los VTC. Oikonomics [en línea]. Mayo 2021, no. 15, pp. 1-7. ISSN: 2339-9546. DOI: https://doi.org/10.7238/o.n15.2106

Los textos publicados en esta revista están sujetos -si no se indica lo contrario- a una licencia de Reconocimiento 4.0 Internacional de Creative Commons. Puede copiarlos, distribuirlos, comunicarlos públicamente, hacer obras derivadas siempre que reconozca los créditos de las obras (autoría, nombre de la revista, institución editora) de la manera especificada por los autores o por la revista. La licencia completa se puede consultar en https://creativecommons.org/licenses/by/4.0/deed.es_ES.
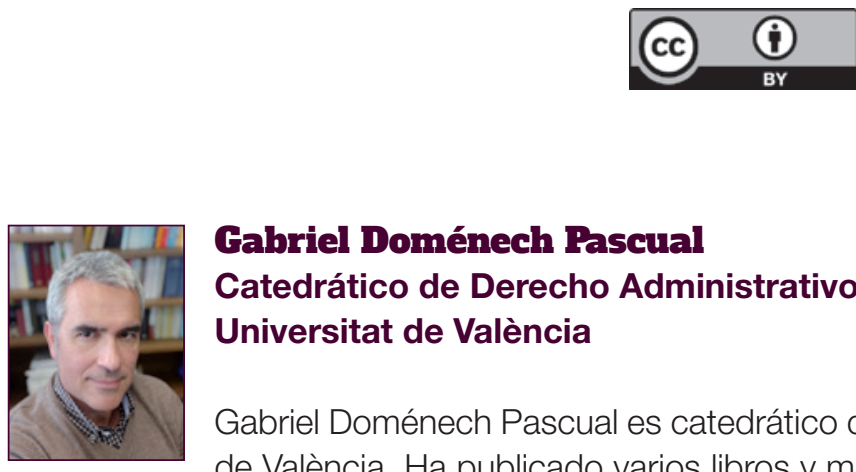

\section{Gabriel Doménech Pascual Catedrático de Derecho Administrativo Universitat de València}

Gabriel Doménech Pascual es catedrático de Derecho Administrativo en la Universitat de València. Ha publicado varios libros y más de cien artículos y capítulos sobre muy diversos temas: la invalidez de las normas jurídicas, las obligaciones positivas del Estado de proteger los derechos fundamentales, el régimen jurídico del bienestar animal, la responsabilidad civil del Estado, la llamada economía colaborativa (principalmente, en los sectores del transporte urbano y las viviendas de uso turístico), las tasas judiciales, los sistemas de retribuciones variables de los jueces, la especialización judicial, etc. Desde hace unos años viene orientando su actividad investigadora y docente principalmente hacia el análisis económico del derecho público. 